\title{
Smoking is associated with constant cough which is resistant to oseltamivir therapy for suspected COVID-19
}

Satoru Chiba ( $\nabla$ chibas_0317@yahoo.co.jp )

Sapporo Suzuki Hospital

\section{Research Article}

Keywords: smoking, cough, COVID-19, oseltamivir therapy

Posted Date: May 17th, 2021

DOI: https://doi.org/10.21203/rs.3.rs-287518/v2

License: (c) (i) This work is licensed under a Creative Commons Attribution 4.0 International License.

Read Full License 


\section{Abstract}

Smoking depresses pulmonary immune function and is a risk factor contracting more serious outcomes among people who become infected. The association between smoking and persistent respiratory symptoms which are resistant to oseltamivir for suspected coronavirus disease 2019 (COVID-19) was evaluated. A total of 22 COVID-19 suspected medical personnel and their cohabitation families without hypoxia and lung diseases, who came to the adult fever clinic were studied. All patients received oseltamivir and antibacterial therapy together. The median age was 40 years. The study patients were divided into 2 groups; individuals who currently smoke in 11 patients (current smokers) and never smoked in 11 (never smokers). Four (36\%) of 11 current smokers experienced constant cough even after oseltamivir administration, compared with $0 \%$ of never smokers $(\chi 2=4.90 ; p<0.05)$. The cough score decreased from $1.5 \pm 0.5$ at baseline to $0.3 \pm 0.5$ during the one week after ciclesonide administration $(p<0.001)$ and constant cough disappeared in three cases out of four current smokers. Because current smokers are at a higher risk to develop constant cough which is resistant to oseltamivir therapy for suspected COVID-19 as compared to never smokers, smoking cessation should be recommended at an early stage. Ciclesonide may improve the constant cough in suspected COVID-19.

\section{Main Text}

In late December 2019, an outbreak of an emerging coronavirus disease 2019 (COVID-19) due to severe acute respiratory syndrome coronavirus 2 (SARS-CoV-2) started in Wuhan, China and rapidly spread in China and outside ${ }^{1}$. The World Health Organization (WHO) declared the epidemic of COVID-19 as a pandemic on 12 March $2020^{2}$. In the early stage of an infection with SARS-CoV-2, the most prevalent symptoms are fever and acute cough ${ }^{3}$. I reported that oseltamivir helped improve not only fever but also acute cough in the early stage of suspected COVID-19 ${ }^{4}$. But part of patients had constant cough and sputum production after oseltamivir treatment. For such "oseltamivir resistant cases", an effective new therapy is urgently required.

An elderly COVID-19 patient with underlying disease is the typical patient that may experience a severe disease course ${ }^{5}$. Elderly patients also have an immune system that does not respond well enough to fight an infectious agent. In addition, most of the elderly patients have other comorbidities such as diabetes mellitus, chronic obstructive pulmonary disease, cardiovascular disease, etc. Under these circumstances, the already deteriorated immune system tries to defend itself against these diseases by further reducing its overall activity and capability of fighting an infection. Smoking depresses pulmonary immune function and causes chronic inflammation in the lungs, so the immune system is constantly defending itself against chronic inflammation. COVID-19 is more likely to deteriorate due to an increase in comorbidities in elderly, which may lead to immune dysfunction in elderly COVID-19 patients, and the defense mechanisms are impaired, so they may develop life-threatening complications ${ }^{6}$. The WHO confirmed that smokers may experience severe complications from COVID-19, compared with never 
smokers ${ }^{7}$. However, the influence of smoking behavior on the respiratory symptoms of COVID-19 has not been investigated sufficiently.

The aim of this study was to investigate the association between smoking and persistent respiratory symptoms which are resistant to oseltamivir for suspected COVID-19.

\section{Results}

\section{Patients characteristics}

The study population included 22 patients meeting the inclusion criteria in this study who had at least 7 days of follow-up at the time of the present analysis. The baseline characteristics of the whole population are shown in Table 1. Overall, 13 patients were female (59\%). Median age was 40 years (interquartile range [IQR] 25-47 years). Only one patient was elderly (>65 years of age). One patient was obese [body mass index $(\mathrm{BMI})>30$ ]. All participants were negative with a local diagnostic test for influenza, of which $22(100 \%)$ were using rapid antigen assays. Half of patients were current smokers [11 (50\%)]. Less than half had underlying comorbidities [9 (41\%)], including dyslipidemia [6 (27\%)], hypertension [4 (18\%)], and diabetes [2 (9\%)]. None of the patients had cardiovascular diseases, lung diseases, chronic kidney diseases, cancer, or gastroesophageal reflux disease.

The influenza has not been spread during the study in Sapporo and the all number of influenza patient reports per fixed point of the coverage period was almost less than 1.00, although the number was 1.61 only in the period from 9 March 2020 to 15 March 2020. But the COVID-19 epidemic has been going around during the study in Sapporo. The Governor of Hokkaido announced the Declaration of a New Coronavirus Emergency on 28 February 2020, calling on locals to refrain from going out. The Japanese Prime Minister expanded the state of emergency declaration to include every prefecture within the country on 16 April 2020. Sapporo-city in Hokkaido has one of the highest incidences of COVID-19 infections in Japan, with the number of cases at 1232 as of 14 September 2020, although the polymerase chain reaction (PCR) test for SARS-CoV-2 was of narrow application in Japan. Many people could have been unknowingly positive for SARS-CoV-2 in Sapporo.

\section{Effect of oseltamivir}

All patients received antiviral therapy [oseltamivir, $22(100 \%)]$. In addition, all patients also received antibacterial therapy for seven days: levofloxacin [19 (86\%)], garenoxacin [2 (9\%)], or amoxicillin/clavulanic acid [1 (5\%)], considering the possibility of secondary infections with bacteria.

The symptoms of baseline and after oseltamivir treatment are shown in Table 2. The major symptoms at onset of illness included fever [22 (100\%)], sore throat [19 (86\%)], myalgia and fatigue [17 (77\%)], and cough [11 (50\%)]. Less common symptoms included headache [10 (45\%)], chills [6 (27\%)], diarrhea[4 (18\%)], and sputum production[3 (14\%)]. None of the patients had hair loss. No patient had olfactory and taste disorders. Oseltamivir helped improve fever as well as sore throat in this study. 
The study was divided into two groups; individuals who currently smoke in 11 patients (current smokers) and never smoked in 11 (never smokers). Never smokers were younger on average than current smokers $(\mathrm{p}<0.01)$. There were no differences in sex, $\mathrm{BMI}$, and $\mathrm{SpO} 2$ between the two groups. The peak temperature was significantly lower in current smokers than in never smokers $\left(37.7 \pm 0.2^{\circ} \mathrm{C}\right.$ versus $38.4 \pm 0.8^{\circ} \mathrm{C}$; $p<$ $0.01)$. Cough significantly decreased only in never smokers after oseltamivir administration $(p<0.05)$. Four of 11 current smokers (36\%) experienced constant cough, compared with $0 \%$ of never smokers $\left(\chi^{2}=4.90 ; p<0.05\right.$, Table 3$)$.

\section{Effect of ciclesonide}

Only four patients in current smokers had a constant cough despite oseltamivir administration. Three patients out of four were female (75\%). Median age of four patients was 55 years (IQR 46-63 years). No patient was elderly. Most patients had underlying diseases [3 (75\%)], including dyslipidemia [3 (75\%)], hypertension [1 (25\%)], and diabetes [1 (25\%)]. One patient was obese. Three patients out of four presented with sputum production as well as cough several days after symptom onset, despite oseltamivir administration. Because our hospital was unable to provide a respirator, it was felt to be great dangerous in having patients with worsening respiratory symptoms. Ciclesonide was administered 6-10 days after onset of respiratory symptoms or fever because of constant cough.

At the start of ciclesonide, there was no fever above $37.2^{\circ} \mathrm{C}$ and SpO2 could be maintained at $96 \%$ or more with room air in four patients. We let 3 medical personnel come back 4-7 days after ciclesonide initiation, because their symptoms completely disappeared. And the sputum also disappeared in these 2 patients. Another patient in her 40s without underlying diseases and obesity had attained slight reductions 7 days after ciclesonide initiation, but her cough grew worse after ciclesonide was discontinued and two inhales of fluticasone propionate / formoterol fumarate dehydrate $(375 / 15 \mu \mathrm{g})$ at one time, twice daily were administered. And her sputum production also persisted. Her chest computed tomography did not show any pneumonia. Overall, ciclesonide were well tolerated. None of the participating subjects developed an acute exacerbation during the trial period. After ciclesonide administration, the cough score decreased from $1.5 \pm 0.5$ at baseline to $0.3 \pm 0.5$ during the one week of treatment $(p<0.001)$.

\section{Discussion}

Early oseltamivir administration improved acute cough as well as fever in COVID-19 suspected outpatients without hypoxia when it is used in combination with antibacterial therapy ${ }^{4}$. However, there are occasionally patients, especially smokers, whose cough and sputum production do not sufficiently decrease after oseltamivir therapy. This study suggests that active smoking is linked with the persistent respiratory symptoms in suspected COVID-19. The finding that smoking is associated with COVID-19 progression is not surprising because of the adverse effects of smoking on pulmonary immune function 11,12 . Because COVID-19 can predominantly damage the lungs, smoking cessation should be 
encouraged. In addition, the viral entry has been shown to cause cytokine storm involving excessive production of proinflammatory cytokines including interleukin (IL)-6, which is augmented by smoking.

Shin et al. made a simple hypothetical model of COVID-19 for the inflammatory processes and cytokine storm, an immune inflammatory overreaction. First, cytokine storm may start from the early course of disease to later stage according to the degree of activation of host inflammatory response and it may be rapidly progressive at any time. Second, there may be no correlation between clinical and pathologic views ${ }^{13}$. The patient may be non-symptomatic or have mild symptoms despite severely progressed conditions, which will delay the appropriate treatment at an early stage. If the COVID-19 symptoms are severe, the patients are hospitalized and the treatment is done, though they have not been established yet. However, no treatment is provided for the patients with mild symptoms who are isolated at home or shelter. Among them, there will be also some cases in whom cytokine storm has progressed ${ }^{14}$, leading to sudden death. I guess that the constant cough in current smokers after oseltamivir administration means a respiratory symptom at an early stage of cytokine storm. Active smoking could trigger off cytokine storm secondary to COVID-19.

The increase in various proinflammatory cytokines induced by COVID-19 might be suppressed by short course of low dose steroids in an early stage of disease. But steroids may not be effective in a later stage of disease such as cytokine storm even though high dose of steroids are used. It is to be desired that the treatment should be initiated before COVID-19 pneumonia with hypoxia appears, and then it is expected to have the effect of improving the respiratory symptoms and preventing progression to severe pneumonia. In addition, there are limited evidences from case reports suggesting that ciclesonide prevents progression to severe pneumonia when administered in the middle stage of CIVID-19 ${ }^{15}$. In the present study, COVID-19-suspected patients in oseltamivir resistant cases took inhaled ciclesonide and their respiratory symptoms improved, although the possibility that this would have been the natural course of the disease in these patients cannot be excluded. Therefore, the inhalation of ciclesonide is a potentially useful new therapeutic strategy in COVID-19 cases. Systemic steroid treatment is controversial in COVID-19 due to the possibility of prolonging viremia and complications such as secondary infection ${ }^{16}$, although systemic administration of dexamethasone is being considered in later stage ${ }^{17}$. Ciclesonide is a safe drug that is widely used as a steroid for inhalation from premature babies and newborns to the elderly and is said to be effective in controlling chronic inflammation of the respiratory tract. Ciclesonide is an inhaled prodrug that stays on the lung surface with only minor increases in blood levels. In a report published on 12 March 2020, they showed that while fluticasone demonstrated no antiviral effects, ciclesonide almost completely suppressed the growth of SARS-CoV-2 at low concentration ${ }^{18}$. Ciclesonide seemed to be more effective on constant cough than fluticasone that had an accompanying long-acting beta-agonist in a ciclesonide resistant case of this study. In addition, ciclesonide has anti-inflammatory effects that suppress the inflammatory mediators, such as IL-6 released from lung epithelial cells, thereby reducing cytokine storm. 
Until now, no specific treatment has been recommended for COVID-19. But the consensus on the management of COVID-19 infected patients is moving toward the need of a biphasic approach. During the first phase of the disease (from onset of the symptoms up to 7 days) antiviral therapy could be considered. In the second inflammatory phase of the disease, the use of anti-inflammatory therapy such as with steroids or cytokine inhibitors may be useful ${ }^{19}$. Once, cytokine storm occur, antiviral treatment alone is not enough and should be combined with appropriate anti-inflammatory treatment. Early recognition and appropriate treatment of cytokine storm will decrease the mortality in COVID-19. I provide a simplified approach through two steps. Early oseltamivir administration helps improve fever in the first phase. In addition to antivirals, current smokers should quit smoking immediately. In the second phase, a ciclesonide inhalation may be considered, if cough persists even after oseltamivir administration. The presence of constant cough in oseltamivir resistant cases may select for a population with a greater likelihood to benefit from ciclesonide.

\section{Limitations of the study}

This study has some limitations; however, in the current context, it is believed that the results should be shared with the scientific community. First, the number of patients was too small to draw definite conclusions. Further studies are clearly needed. Second, there was no control group for patients without use of ciclesonide, therefore, it is difficult to directly compare constant cough in patients with and without use of ciclesonide. Third, this study was performed in Japan, a country that the test for SARS-CoV-2 could only be performed if pneumonia was present or a confirmed close contact with a SARS-CoV-2 patient in the beginning. Therefore, there was a lack of coronavirus tests in this study and patients with other influenza-like respiratory diseases may have been included, though COVID-19 has been spreading during the study in Sapporo. Fourth, a definitive diagnosis of influenza may also require a PCR test in general. However, influenza diagnosis information was obtained through use of rapid diagnosis kits by medical institution and the sensitivity of the influenza antigen test is equal to that of the PCR test for SARS-CoV-2. Therefore, it is believed that this diagnosis information was reliable.

\section{Conclusions}

This study demonstrated that active smoking is associated with persistent respiratory symptoms after oseltamivir administration in suspected COVID-19, and smoking cessation may help in improving outcomes. In addition, the inhalation of ciclesonide has an excellent therapeutic potential in patients with constant cough at an early course of suspected COVID-19. Large scale controlled studies should therefore be performed to establish the efficacy of ciclesonide on the long-term prognosis in COVID-19 patients without hypoxia which does not sufficiently respond to oseltamivir therapy.

\section{Methods}

\section{Study Design and Participants}


A total of 34 medical personnel and their cohabitation families with medication in the fever clinic, from 1 March 2020, to 14 September 2020 were prospectively enrolled in the study if they fulfilled five primary criteria: i) age $\geqq 20$ years, ii) peak temperature $\left(\geqq 37.5^{\circ} \mathrm{C}\right)$, iii) one or more respiratory symptoms; iv) a nose swab for influenza A and B was negative, v) peripheral capillary oxygen saturation (SpO2) $\geqq 96 \%$ (Fig. 1). Exclusion criteria were as follows: 1 ) history of asthma; 2 ) incidences of self-reported wheeze, pharmacy data indicating asthma-like symptoms or variability in lung function in the previous year ${ }^{8} ; 3$ ) current treatment that might influence the cough; 4) any concurrent airway disease (e.g. pneumonia, cancer, tuberculosis); and 5) uncontrolled systemic disease or pregnancy. Nine with low grade fever were excluded. Three patients with hypoxia and lung diseases were excluded. Thus, the study population consisted of 22 patients without low grade fever, hypoxia, and lung diseases. Eligible participants to receive oseltamivir $75 \mathrm{mg}$ were given orally twice a day for 5 days.

A small series of four consecutive suspected COVID-19 patients whose cough did not decrease even after the administration of oseltamivir therapy received inhalation of ciclesonide. Daily inhalation of ciclesonide [Alvesco Inhalation $200 \mu \mathrm{g}$ inhaler (56 puffs/kit); Teijin, Tokyo, Japan], $800 \mu \mathrm{g} /$ day, was administered to the patients. Either two puffs of $200 \mu \mathrm{g}$ metered-dose inhaler (MDI) of open-label ciclesonide in the morning and the evening for a total of 7 days.

\section{Case Definition and Variable Measurement}

A suspected case of COVID-19 was defined as an individual who had an influenza-like illness, as shown by peak temperature $\geqq 37.5^{\circ} \mathrm{C}$, one or more respiratory symptoms (cough, sputum, or sore throat), and a nose swab for influenza was negative. Influenza antigen tests were performed immediately after sample collection. This antigen test ImunoAce Flu is licensed for the diagnosis of human influenza $A$ and $B$ (Tauns Laboratories, Shizuoka, Japan) and has a sensitivity of $67 \%{ }^{9}$.

Patients completed a daily diary card for cough (score $0=$ absent, $1=$ mild, $2=$ moderate, $3=$ severe) ${ }^{10}$ and other respiratory tract symptoms regarding the previous day and night. The primary outcome measure was the decrease in daily cough score during the 1 week treatment period. Patients were shown how to complete the daily record card for the symptoms: cough, sputum production, wheezing, shortness of breath, and chest tightness; the scores were $0=$ no symptoms ranging to $3=$ severe symptoms. This was to be completed every morning and every evening during the 1 week of treatment. Patients with smoking history were identified. For patients with smoking history, the amount of smoking, the years of smoking history, and the years of smoking cessation were individually collected.

\section{Statistical Analysis}

Data are expressed as mean \pm standard deviation or percentages of patients. Differences were compared by Wilcoxon non-parametric test for Gaussian variable. The ratio between baseline and after medication was compared by $\chi^{2}$ test. Differences between the two groups were evaluated using the $\chi^{2}$ test for $2 \times 2$ contingency tables. $p<0.05$ was considered significant for all tests. 


\section{Declarations}

\section{Ethics approval and consent to participate}

All procedures performed in studies involving human participants were in accordance with the ethical standards of the institutional and/or national research committee at which the studies were conducted (Sapporo Suzuki Hospital; IRB Approval Number 2020-002, 1 March 2020) and with the 1964 Helsinki declaration and its later amendments or comparable ethical standards. Written informed consent was obtained from each study patient. Before analyzing the data all identifiable information was deleted and specific individuals cannot be identified by these data. Therefore, this was approved by the ethics committee.

\section{Consent for publication}

Not applicable.

\section{Data Availability}

Not applicable.

\section{Acknowledgments}

The author thanks all of the staff members and their cohabitation families of the Sapporo Suzuki Hospital who agreed to participate in this study, the director, the chairman, the assistant directors, the other doctors and all the nurses who cared for these patients and all the technical and paramedical staff of the hospitalization units for their support in this difficult context.

\section{Author Contributions}

S.C. analyzed, interpreted the patient data, wrote the manuscript text, and prepared figures 1 and table 13. The author read and approved the final manuscript.

\section{Funding}

Not applicable.

\section{Additional Information}

Competing interests: The author declares that I have no competing interests, including financial and nonfinancial interests.

\section{Abbreviations}

BMI: body mass index. Sp02: peripheral capillary oxygen saturation. BCG: Bacille de Calmette et Guérin. 


\section{References}

1 Lai, C.C., Shih, T.P., Ko, W.C., Tang, H.J., Hsueh, P.R. Severe acute respiratory syndrome coronavirus 2 (SARS-CoV-2) and coronavirus disease-2019 (COVID-19): the epidemic and the challenges. Int $J$ Antimicrob Agents. 55, 105924 (2020).

2 WHO. Director-General's opening remarks at the media briefing on COVID-19-11 March. 2020. https://www. who.int/director-general/speeches/detail/who-directorgeneral-s-opening-remarks-at-themedia-briefing-oncovid-19--11-march-2020.

3 Zhang, J.J. et al. Clinical characteristics of 140 patients infected with SARS-CoV-2 in Wuhan, China. Allergy. 75, 1730-1741. doi: 10.1111/all.14238 (2020).

4 Chiba, S. Effect of early oseltamivir on outpatients without hypoxia with suspected COVID-19. Wien Klin Wochenschr. 133, 292-297. doi: 10.1007/s00508-020-01780-0 (2021).

5 Wang, L. et al. Coronavirus disease 2019 in elderly patients: characteristics and prognostic factors based on 4-week follow-up. J Infect. 80, 639-645. doi: 10.1016/j.jinf.2020.03.019 (2020).

6 Pelechas, E., Drossou, V., Voulgari, P.V., Drosos, A.A. Anti-rheumatic drugs for the fight against the novel coronavirus infection (SARS-CoV-2): what is the evidence? Mediterr J Rheumatol. 31(Suppl 2), 259-267. doi: 10.31138/mjr.31.3.259 (2020).

7 WHO Statement: Tobacco Use and COVID-19. Available online: https://www.who.int/news/item/11-052020-who-statement-tobacco-use-and-covid-19

8 Tattersfield, A.E., Knox, A.J., Britton, J.R., Hall, I.P. Asthma. Lancet. 360, 1313-22. doi: 10.1016/s01406736(02)11312-2 (2002).

9 Yamanaka, T. et al. Evaluation of twenty-two rapid antigen detection tests in the diagnosis of equine influenza caused by viruses of H3N8 subtype. Influenza Other Respir Viruses. 10, 127-33 (2016).

10 Ponsioen, B.P., Hop, W.C., Vermue, N.A., Dekhuijzen, P.N., Bohnen, A.M. Efficacy of fluticasone on cough: a randomised controlled trial. Eur Respir J. 25, 147-52. doi: 10.1183/09031936.04.00053604 (2005).

11 Arcavi, L., Benowitz, N.L. Cigarette smoking and infection. Arch Intern Med. 164, 2206-16. doi: 10.1001/archinte.164.20.2206 (2004).

12 Bauer, C.M.T., Morissette, M.C., Stämpfli, M.R. The influence of cigarette smoking on viral infections: translating bench science to impact COPD pathogenesis and acute exacerbations of COPD clinically. Chest 143, 196-206. doi: 10.1378/chest.12-0930 (2013). 
13 Kronbichler, A., Effenberger, M., Eisenhut, M., Lee, K.H., Shin, J.I. Seven recommendations to rescue the patients and reduce the mortality from COVID-19 infection: an immunological point of view. Autoimmun Rev. 19, 102570. doi: 10.1016/j.autrev.2020.102570 (2020).

$14 \mathrm{Shi}, \mathrm{H}$. et al. Radiological findings from 81 patients with COVID-19 pneumonia in Wuhan, China: a descriptive study. Lancet Infect Dis. 20, 425-434. doi: 10.1016/S1473-3099(20)30086-4 (2020).

15 Iwabuchi, K. et al. Therapeutic potential of ciclesonide inahalation for COVID-19 pneumonia: report of three cases. J Infect Chemother. 26, 625-632. doi: 10.1016/j.jiac.2020.04.007 (2020).

16 Russell, C.D., Millar, J.E., Baillie, J.K. Clinical evidence does not support corticosteroid treatment for 2019-nCoV lung injury. Lancet. 395, 473-475. doi: 10.1016/S0140 6736(20)30317-2 (2020).

17 RECOVERY Collaborative Group, Horby, P. et al. Dexamethasone in hospitalized patients with Covid19-preliminary report. N Eng/ J Med. 384, 693-704. doi10.1056/NEJMoa2021436 (2021).

18 Matsuyama, S. et al. The inhaled steroid ciclesonide blocks SARS-CoV-2 RNA replication by targeting the viral replication-transcription complex in cultured cells. J Virol. 95, e01648 20. doi:

10.1128/JVI.01648-20 (2020).

19 Alberici, F. et al. Management of patients on dialysis and with kidney transplant during SARS-COV-2 (COVID-19) pandemic In Brescia, Italy. Kidney Int Rep. 5, 580-5 (2020).

\section{Tables}

Table 1. Patient characteristics 


\begin{tabular}{|c|c|c|c|}
\hline Variables & Current Smokers $(n=11)$ & $\begin{array}{l}\text { Never Smokers } \\
\qquad(\mathrm{n}=11)\end{array}$ & $\mathrm{P}$ value \\
\hline Age, years & $47 \pm 14$ & $31 \pm 10$ & $<0.01$ \\
\hline \multicolumn{4}{|l|}{ Sex } \\
\hline Female, n(\%) & $7(64 \%)$ & $6(55 \%)$ & 0.68 \\
\hline BMI $\left(\mathrm{kg} / \mathrm{m}^{2}\right)$ & $25 \pm 7$ & $22 \pm 3$ & 0.23 \\
\hline SpO2 (\%) & $98 \pm 1$ & $98 \pm 1$ & 0.50 \\
\hline BCG, n(\%) & $10(91 \%)$ & $11(100 \%)$ & 0.33 \\
\hline \multicolumn{4}{|l|}{ Basic disease } \\
\hline Hypertension, n(\%) & $2(18 \%)$ & $2(18 \%)$ & 1.00 \\
\hline Diabetes mellitus, n(\%) & $2(18 \%)$ & $0(0 \%)$ & 0.15 \\
\hline Dyslipidemia, n(\%) & $5(45 \%)$ & $1(9 \%)$ & 0.06 \\
\hline \multicolumn{4}{|l|}{ Medications } \\
\hline Oseltamivir, n(\%) & $11(100 \%)$ & $11(100 \%)$ & प \\
\hline Levofloxacin, n(\%) & $10(91 \%)$ & $9(82 \%)$ & $\square$ \\
\hline Garenoxacin, n(\%) & $0(0 \%)$ & $2(18 \%)$ & प \\
\hline Amoxicillin/clavulanic acid, n(\%) & $1(8 \%)$ & $0(0 \%)$ & $\square$ \\
\hline Ciclesonide, n(\%) & $4(36 \%)$ & $0(0 \%)$ & ( \\
\hline
\end{tabular}

BMI: body mass index. SpO2: peripheral capillary oxygen saturation. BCG: Bacille de Calmette et Guérin.

Table 2. COVID-19-suspected signs and symptoms of baseline and after oseltamivir treatment 


\begin{tabular}{lcccccc}
\hline & \multicolumn{2}{c}{ Current Smokers (n=11) } & \multicolumn{2}{c}{ Never Smokers (n=11) } \\
& Baseline & 7 days & P value & Baseline & 7 days & P value \\
\hline Fever, n(\%) & $11(100 \%)$ & $2(18 \%)$ & $<0.001$ & $11(100 \%)$ & $1(9 \%)$ & $<0.001$ \\
Myalgia or fatigue, n(\%) & $7(64 \%)$ & $1(9 \%)$ & $<0.01$ & $10(91 \%)$ & $0(0 \%)$ & $<0.001$ \\
\hline Sore throat, n(\%) & $10(91 \%)$ & $2(18 \%)$ & $<0.001$ & $9(82 \%)$ & $1(9 \%)$ & $<0.001$ \\
\hline Cough, n(\%) & $7(64 \%)$ & $4(36 \%)$ & 0.19 & $4(36 \%)$ & $0(0 \%)$ & 0.04 \\
\hline Chills, n(\%) & $2(18 \%)$ & $0(0 \%)$ & 0.17 & $4(36 \%)$ & $0(0 \%)$ & 0.04 \\
\hline Headache, n(\%) & $6(55 \%)$ & $0(0 \%)$ & $<0.01$ & $4(36 \%)$ & $0(0 \%)$ & 0.04 \\
\hline Diarrhea, n(\%) & $3(27 \%)$ & $2(18 \%)$ & 0.59 & $1(9 \%)$ & $0(0 \%)$ & 0.34 \\
\hline Sputum production, n(\%) & $1(9 \%)$ & $4(36 \%)$ & 0.08 & $2(18 \%)$ & $1(9 \%)$ & 0.59 \\
\hline Dyspnea, n(\%) & $1(9 \%)$ & $0(0 \%)$ & 0.34 & $1(9 \%)$ & $0(0 \%)$ & 0.34 \\
\hline Appetite loss, n(\%) & $0(0 \%)$ & $2(18 \%)$ & 0.17 & $4(36 \%)$ & $0(0 \%)$ & 0.04 \\
\hline Nausea or vomit, n(\%) & $1(9 \%)$ & $1(9 \%)$ & 1.00 & $1(9 \%)$ & $0(0 \%)$ & 0.34 \\
\hline
\end{tabular}

Table 3. Smoke History and cough 7 days after oseltamivir treatment

\begin{tabular}{lccc}
\hline Group & \multicolumn{2}{c}{ Symptoms after oseltamivir therapy } & Row total \\
\cline { 2 - 3 } & Constant cough & No cough & \\
\cline { 2 - 3 } Current smokers & 4 & 7 & 11 \\
Never smokers & 0 & 11 & 11 \\
\hline Column total & 4 & 18 & 22 \\
\hline
\end{tabular}

Figures 
Figure 1

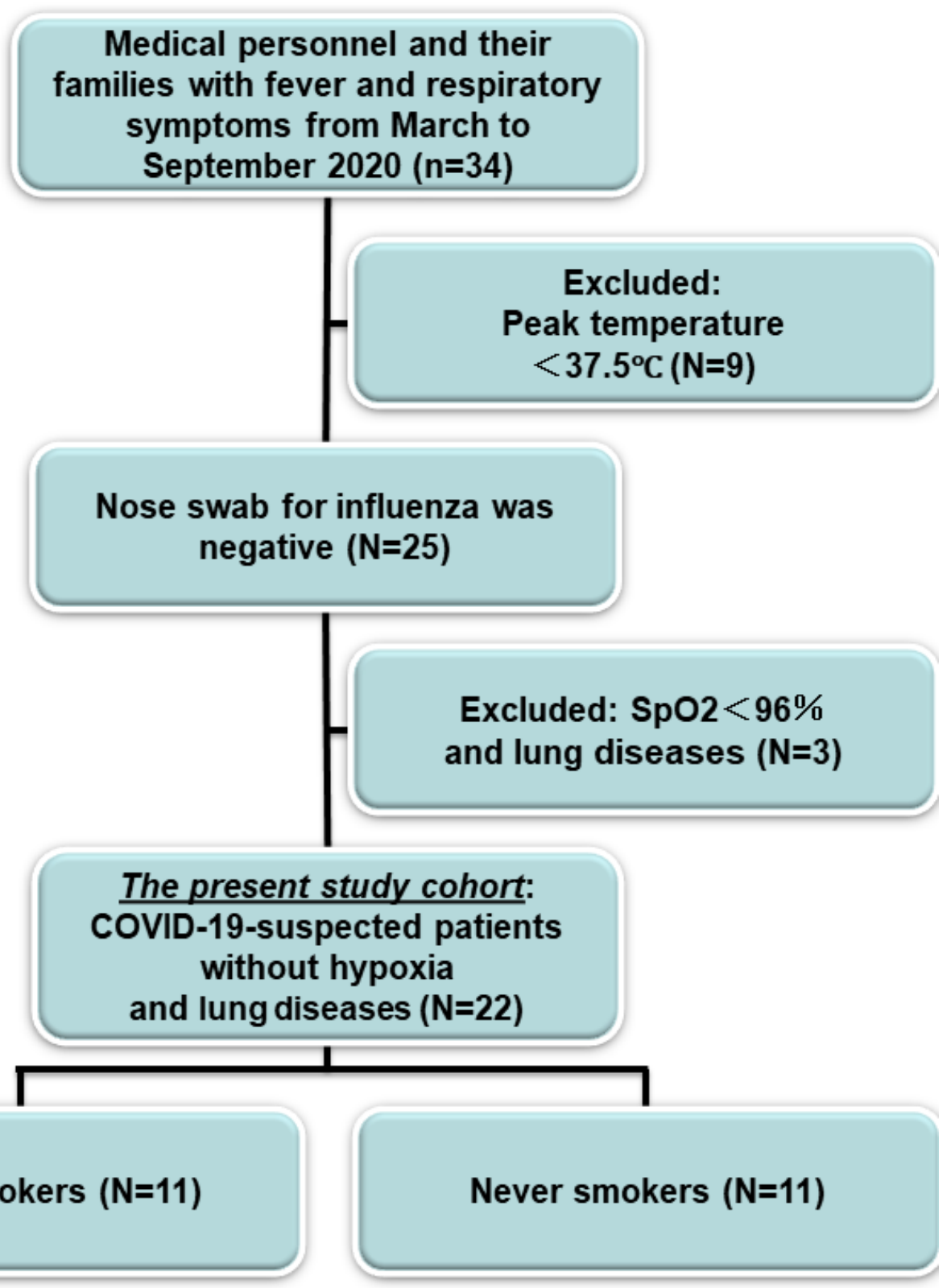

Figure 1

Flow chart of the COVID-19-suspected outpatients study cohort Sp02: peripheral capillary oxygen saturation 